\title{
Maximum-Likelihood-Based Filtering for Attitude Determination via GPS Carrier Phase
}

\author{
H. M. Peng, Y. T. Chiang and F. R. Chang \\ Department of Electrical Engineering \\ National Taiwan University, Taipei, Taiwan, R. O. C. \\ L. S. Wang \\ Institute of Applied Mechanics \\ National Taiwan University, Taipei, Taiwan, R. O. C.
}

\begin{abstract}
In this paper, a maximum-likelihood-based (MLbased) filter is proposed for attitude determination via the GPS carrier phase observables. The quaternion representation is adopted here to describe the attitude. Hence, the norm constraint on the quaternion should be considered. The ML estimation with Lagrange multipliers can be used to consider simultaneously the evolution equation and the constraint, and to minimize the error covariance matrix. The attitude determination via GPS carrier phase observables is fulfilled in two steps. The first step is the GPS carrier phase ambiguity resolution. After the integer ambiguities being fixed, the ML-based filter is used to determine the optimal attitude. The advantage of adopting the quaternion as the state vector to describe the kinematic behaviors is that no singular problems arise. To verify our algorithm, the simulation has been conducted. In the simulation, the white noises are added on the carrier phase observables to assess the performance of the proposed method. The body frame is formed by three non-colinear GPS antennae which are mounted on a platform with two aluminum bars representing the baseline vectors. According to the simulation, our method is sound and effective.
\end{abstract}

\section{INTRODUCTION}

The problems of attitude determination frequently arise, such as in the navigation and control of vehicles, in the attitude determination and control of spacecrafts. The sun sensor, the horizon sensor, and the gyroscope are some popular sensors [20] to obtain the attitude information. All these sensors have some defects, however. The sun sensor is invalid when the satellite is behind the Earth. Since the horizon of the Earth is poor defined, the accuracy of the horizon sensor is limited. The drift problem, i.e., the accumulation of errors, associated with the gyroscope makes it necessary to calibrate periodically. The advent of GPS provides another route to solve the problem of attitude determination. According to the system characteristics, GPS does not have any of the problems that plague the sensors mentioned above.

GPS is a satellite-based navigation system, and its applications includes the positioning, navigation, tracking, and surveying [7]. Considering the accuracy requirement of attitude determination, the GPS carrier phase observables are the most adequate measurements. Based on the idea of interferometry, several algorithms for attitude determination have been developed in $[2,5,10]$. The main difficulty of using carrier phase measurements is to find the initial ambiguities. This problem has been investigated by many researchers. Various methodologies have been proposed, such as the ambiguity search techniques $[4,10,19]$, the ambiguity function methods [13,21], and the tripledifference methods $[2,14]$. The first two methods process the double differences of carrier phase observables. The integer ambiguity problem is solved by search algorithms either on the space of possible ambiguous values or on the space of possible coordinates. The tripledifference methods including the space-difference and time-difference algorithm eliminate the ambiguities to calculate the coordinates. In this paper, the ambiguity function method is used to estimate the carrier phase integer ambiguities.

On the other hand, an appropriate algorithm for determining the attitude is also essential, which affects the computation efficiency and the accuracy of the attitude determination. The problem of attitude determination is to find an orthogonal matrix, also called as the attitude matrix, which can be used to represent the rotational relationship between the vectors measured in 
the reference frame and in the body frame. The attitude matrix has several representations [17], such as the nine components in a $3 \times 3$ rotation matrix, the Eulerian angles, and the Euler-Rodrigues symmetric parameters (also known as the quaternions). The rotation matrix is the most natural representation, but the orthogonal property is difficult to retain. The Eulerian angles are 3-degree-of-freedom minimal representation without any constraint. However, the singularity may occur at some specific angles. The quaternion represents the attitude by four parameters with one constraint. The QUEST algorithm [18] is one typical approach to solve the attitude determination problem, but it is suitable for single epoch only. Considering the vector measurements at different epochs, some modified algorithms are proposed in the literature, such as the filter QUEST method [15], the REQUEST algorithm [1], the extended Kalman filter method $[8,16]$, and the $H_{\infty}$ filter method [11]. The first two methods are upgraded from the QUEST algorithm, but they can not handle the process noise. Using quaternion as the state variables, the latter two approaches can tackle the process noise and the measurement noise. However, the norm constraint of quaternion is resolved by the dimensional reduction of the state variables. A more typical approach is to consider the evolution equation of the quaternion and the norm constraint equation together. In other words, the differential equation and the algebraic constraint are dealt with simultaneously. In this paper, a maximum-likelihood-based filtering with Lagrange multipliers is adopted for considering such a singular system.

To verify our algorithms, the simulation has been conducted. The simulation is used to assess the performance of the proposed method. Three non-colinear antennae are assumed to be mounted on a two-degree-offreedom platform with two aluminum bars representing the baseline vectors. By processing the generated carrier phase observables with white noises from the three antennae attached to the ends of the bars, the proposed filter can be used to estimate the attitude. The simulation shows that the proposed method is sound and effective.

This paper is organized as follows. In section II, the GPS carrier phase double-difference model is briefly described. The ambiguity function method is also discussed in this section. In section III, the concept of ML estimation with Lagrange multipliers is discussed. The application to attitude determination is introduced in section IV. The simulation is discussed in section V. Conclusions are given in section VI.

\section{GPS Carrier Phase Model}

The GPS carrier phase observable counts the beat phase, i.e., the difference between the L-band carrier wave from a satellite and the reference signal generated by the receiver. It can be modeled as [6]

$$
\phi_{A}^{j}=\rho_{A}^{j}+c\left(\delta t^{j}-\delta t_{A}\right)+\lambda N_{A}^{j}-d_{i o n}^{j}+d_{t r o p}^{j}+\varepsilon_{A}^{j},
$$

where $\phi_{A}^{j}$ is the carrier phase measurements of the receiver $A$ from the $j$-th GPS satellite; $\rho_{A}^{j}$ is the true distance between the receiver $A$ and the $j$-th GPS satellite; $c$ is the speed of light; $\delta t^{j}$ and $\delta t_{A}$ are the clock biases of the $j$-th satellite and the receiver $A$, respectively; $\lambda$ is the GPS carrier wavelength; $N$ denotes the initial phase integer ambiguity; and $d_{i o n}^{j}, d_{\text {trop }}^{j}, \varepsilon_{A}^{j}$ and are the ionospheric delay, the tropospheric delay and the unmodelled errors, respectively. The units of all the terms in the equation are meters.

To eliminate the biases and the errors from satellites and receivers, it is customary to perform double differences between two receivers and two satellites. Denoting the two receivers as $A$ and $B$ and the satellites as $j$ and $k$, the double-difference equation is

$$
\phi_{A B}^{j k}=\rho_{A B}^{j k}+\lambda N_{A B}^{j k}+\varepsilon_{A B}^{j k},
$$

where the convention $*_{A B}^{j k}=\left(*_{B}^{k}-*_{A}^{k}\right)-\left(*_{B}^{j}-*_{A}^{j}\right)$ is used, and the asterisk may be replaced by $\phi, \rho, N$ and $\varepsilon$. The satellite clock bias, the receiver clock bias, the ionospheric delay, and the tropospheric delay are then eliminated in the double-difference model.

The concept of interferometry is described in the following. In Figure $1, R_{A}$ and $R_{B}$ denote two GPS antennae which are mounted on the two ends of baseline vector a, respectively. They receive signals from the same GPS satellite denoted as $S^{j}$ with the same unit directional vector $\mathbf{g}^{j}$. The difference between the distances from the two antennae to the satellite $S^{j}$ is then

$$
\rho_{A B}=\left(\mathbf{g}^{j}\right)^{T} \mathbf{a},
$$

where the superscript $T$ denotes the transpose.

With $n$ satellites $(n \geq 4)$ being observed at the same time, the matrix form of the equations can be written from (2) and (3) as

$$
\mathbf{\Phi}=\mathbf{S}^{T} \mathbf{a}+\lambda \mathbf{N}+\mathbf{E},
$$

where

$$
\begin{aligned}
\mathbf{S} & =\left[\begin{array}{llll}
\mathbf{s}^{1} & \mathbf{s}^{2} & \cdots & \mathbf{s}^{n-1}
\end{array}\right] \\
& =\left[\begin{array}{llll}
\mathbf{g}^{2}-\mathbf{g}^{1} & \mathbf{g}^{3}-\mathbf{g}^{1} & \cdots & \mathbf{g}^{n}-\mathbf{g}^{1}
\end{array}\right], \\
\mathbf{\Phi} & =\left[\begin{array}{llll}
\phi_{A B}^{12} & \phi_{A B}^{13} & \cdots & \phi_{A B}^{1 n}
\end{array}\right]^{T}, \\
\mathbf{N} & =\left[\begin{array}{llll}
N_{A B}^{12} & N_{A B}^{13} & \cdots & N_{A B}^{1 n}
\end{array}\right]^{T}, \\
\mathbf{E} & =\left[\begin{array}{llll}
\varepsilon_{A B}^{12} & \varepsilon_{A B}^{13} & \cdots & \varepsilon_{A B}^{1 n}
\end{array}\right]^{T} .
\end{aligned}
$$






Figure 1: Concept of interferometry method

The main difficulty in using this model is on the determination of the initial integer ambiguities $\mathbf{N}$. In the following discussion, the ambiguity function method is briefly described for estimating the integer ambiguities. Assuming that the position of the receiver $A$ is known and $n$ satellites are observed. The coordinates of receiver $B\left(\mathbf{p}_{B}\right)$ are the unknown parameters. Consider the ambiguity function defined below

$$
F\left(\mathbf{p}_{B}\right)=\sum_{k=2}^{n} \cos \left(\frac{2 \pi}{\lambda}\left(\phi_{A B}^{1 k}-\rho_{A B}^{1 k}\right)\right) .
$$

Note that the argument of the cosine function is the difference between the double-difference phase observable and the double-difference true range multiplied by $\frac{2 \pi}{\lambda}$. Comparing with (2), this term is equal to the doubledifference ambiguity multiplied by $2 \pi$. Hence, if the correct coordinates $\mathbf{p}_{B}$ are substituted into the ambiguity function, the function $F$ should be maximized. According to the above statements, the ambiguity function method can be divided into three parts. The first step is the computation of an approximation $\mathbf{p}_{B}$, which can be achieved by the DGPS algorithm, or the triple difference method. Next, the candidates generated by the grid points surrounding the approximation $\mathbf{p}_{B}$ are substituted into the ambiguity function. The maximizer is the optimal estimate $\mathbf{p}_{B}$. The final step is to determine the integer double-difference ambiguities, which can be computed from (4).

\section{INTRODUCTION TO ML-BASED FILTERING}

In this section, a maximum-likelihood-based (MLbased) filtering is described. The derivation of the MLbased filtering was proposed in [12], and the results are presented here for completeness. If the considered system is nonlinear and the extra constraint exists, the linearized system based on the Taylor series expansion with constraint is used in the proposed method. Since the constraint equation may not be linear, the final estimate may be found by iterations.

According to the lemmas proved in [12], it has been shown that previously processed measurements can be included in the form of a aggregated measurement for the variables that have been estimated and the irrelevant variables can be discarded. It constitutes the basis of the recursive procedure of maximum likelihood estimates. Based on the above discussion, the linear MLbased filtering can be derived as follows. Considering the following discrete system equation and measurement equation

$$
\begin{aligned}
\mathbf{x}_{k+1} & =\mathbf{A}_{k} \mathbf{x}_{k}+\mathbf{w}_{k}, \\
\mathbf{y}_{k} & =\mathbf{C}_{k} \mathbf{x}_{k}+\mathbf{v}_{k},
\end{aligned}
$$

where $\mathbf{v}_{k}$ and $\mathbf{w}_{k}$ are zero-mean independent Gaussian noises with covariance matrices $\mathbf{R}_{k}$ and $\mathbf{Q}_{k}$, respectively. Let $\mathbf{P}_{k}$ denote the error covariance associated with the filtered estimate $\hat{\mathbf{x}}_{k}$, with $\hat{\mathbf{x}}_{0}$ and $\mathbf{P}_{0}$ given by the prior distribution for $\mathbf{x}_{0} . \hat{\mathbf{x}}_{k+1}$ and $\mathbf{P}_{k+1}$ are, respectively, equal to the maximum likelihood estimate of $\mathbf{x}_{k+1}$ and its associated estimation error covariance based on the following observations

$$
\begin{aligned}
\mathbf{y}_{k+1} & =\mathbf{C}_{k+1} \mathbf{x}_{k+1}+\mathbf{v}_{k+1}, \\
\mathbf{A}_{k} \hat{\mathbf{x}}_{k} & =\mathbf{x}_{k+1}+\mathbf{A}_{k} \mathbf{r}_{k}-\mathbf{w}_{k},
\end{aligned}
$$

where the aggregated measurement $\hat{\mathbf{x}}_{k}=\mathbf{x}_{k}+\mathrm{r}_{k}$ is used to substitute for the prior measurement of $x_{k}$, in which $\mathbf{r}_{k}$ is a Gaussian random vector, independent of $\mathbf{w}_{k}$, with zero mean and variance $\mathbf{P}_{k}$. Furthermore, the filtered estimate $\hat{\mathbf{x}}_{k+1}$ and the corresponding error covariance $\mathbf{P}_{k+1}$ can be obtained from the following recursions:

$$
\begin{gathered}
\hat{\mathbf{x}}_{k+1}=\left[\begin{array}{lll}
\mathbf{0} & \mathbf{0} & \mathbf{I}
\end{array}\right] \\
{\left[\begin{array}{ccc}
\mathbf{A}_{k} \mathbf{P}_{k} \mathbf{A}_{k}^{T}+\mathbf{Q}_{k} & \mathbf{0} & \mathbf{I} \\
\mathbf{0} & \mathbf{R}_{k+1} & \mathbf{C}_{k+1} \\
\mathbf{I} & \mathbf{C}_{k+1}^{T} & \mathbf{0}
\end{array}\right]^{+}\left[\begin{array}{c}
\mathbf{A}_{k} \hat{\mathbf{x}}_{k} \\
\mathbf{y}_{k+1} \\
\mathbf{0}
\end{array}\right],} \\
\mathbf{P}_{k+1}=-\left[\begin{array}{lll}
\mathbf{0} & \mathbf{0} & \mathbf{I}
\end{array}\right] \\
{\left[\begin{array}{ccc}
\mathbf{A}_{k} \mathbf{P}_{k} \mathbf{A}_{k}^{T}+\mathbf{Q}_{k} & \mathbf{0} & \mathbf{I} \\
\mathbf{0} & \mathbf{R}_{k+1} & \mathbf{C}_{k+1} \\
\mathbf{I} & \mathbf{C}_{k+1}^{T} & \mathbf{0}
\end{array}\right]^{+}\left[\begin{array}{l}
\mathbf{0} \\
\mathbf{0} \\
\mathbf{I}
\end{array}\right],}
\end{gathered}
$$

where $\hat{\mathbf{x}}$ means the estimate of $\mathbf{x}$ and the superscript + represents the pseudo-inverse $[3,9]$. If this matrix is invertible, the pseudo-inverse is equivalent to the inverse operation.

If the considered system is nonlinear, and some extra constraints are exist, the linearized system based on the Taylor series expansion with constraint is adopted 
in the proposed method. Assuming that the nonlinear system with constraint equation is modeled as

$$
\begin{aligned}
\mathbf{x}_{k+1} & =\mathbf{f}_{k}\left(\mathbf{x}_{k}\right)+\mathbf{g}_{k}\left(\mathbf{x}_{k}\right) \mathbf{w}_{k} \\
\mathbf{y}_{k+1} & =\mathbf{h}_{k+1}\left(\mathbf{x}_{k+1}\right)+\mathbf{v}_{k+1} \\
\mathbf{0} & =\mathbf{l}_{k+1}\left(\mathbf{x}_{k+1}\right)
\end{aligned}
$$

The nonlinear function $\mathbf{f}_{k}, \mathbf{g}_{k}, \mathbf{h}_{k+1}$, and $\mathbf{l}_{k+1}$, if sufficiently smooth, can be expanded in Taylor series about $\hat{\mathbf{x}}_{k}$ and $\hat{\mathbf{x}}_{k+1}^{-}$as

$$
\begin{aligned}
\mathbf{f}_{k}\left(\mathbf{x}_{k}\right) & =\mathbf{f}_{k}\left(\hat{\mathbf{x}}_{k}\right)+\mathbf{F}_{k}\left(\mathbf{x}_{k}-\hat{\mathbf{x}}_{k}\right)+\cdots \\
\mathbf{g}_{k}\left(\mathbf{x}_{k}\right) & =\mathbf{g}_{k}\left(\hat{\mathbf{x}}_{k}\right)+\cdots=\mathbf{G}_{k}+\cdots \\
\mathbf{h}_{k+1}\left(\mathbf{x}_{k+1}\right) & =\mathbf{h}_{k+1}\left(\hat{\mathbf{x}}_{k+1}^{-}\right)+\mathbf{H}_{k+1}\left(\mathbf{x}_{k+1}-\hat{\mathbf{x}}_{k+1}^{-}\right)+\cdots \\
\mathbf{0} & =\mathbf{l}_{k+1}\left(\hat{\mathbf{x}}_{k+1}^{-}\right)+\mathbf{L}_{k+1}\left(\mathbf{x}_{k+1}-\hat{\mathbf{x}}_{k+1}^{-}\right)+\cdots,
\end{aligned}
$$

where $\hat{\mathbf{x}}_{k+1}^{-}=\mathbf{f}_{k}\left(\hat{\mathbf{x}}_{k}\right)$. Neglecting higher order terms enables us to approximate the model (12), (13) and (14) as

$$
\begin{aligned}
\mathbf{x}_{k+1} & =\mathbf{F}_{k} \mathbf{x}_{k}+\mathbf{G}_{k} \mathbf{w}_{k}+\mathbf{u}_{k} \\
\mathbf{y}_{k+1} & =\mathbf{H}_{k+1} \mathbf{x}_{k+1}+\mathbf{v}_{k+1}+\mathbf{p}_{k+1} \\
\mathbf{0} & =\mathbf{L}_{k+1} \mathbf{x}_{k+1}+\mathbf{z}_{k+1}
\end{aligned}
$$

where $\mathbf{u}_{k}, \mathbf{p}_{k+1}$, and $\mathbf{z}_{k+1}$ are calculated on line from equations

$$
\begin{aligned}
\mathbf{u}_{k} & =\mathbf{f}_{k}\left(\hat{\mathbf{x}}_{k}\right)-\mathbf{F}_{k} \hat{\mathbf{x}}_{k} \\
\mathbf{p}_{k+1} & =\mathbf{h}_{k+1}\left(\hat{\mathbf{x}}_{k+1}^{-}\right)-\mathbf{H}_{k+1} \hat{\mathbf{x}}_{k+1}^{-} \\
\mathbf{z}_{k+1} & =\mathbf{l}_{k+1}\left(\hat{\mathbf{x}}_{k+1}^{-}\right)-\mathbf{L}_{k+1} \hat{\mathbf{x}}_{k+1}^{-} .
\end{aligned}
$$

Thus, the estimate $\hat{\mathbf{x}}_{k+1}$ and the corresponding covariance matrix $\mathbf{P}_{k+1}$ are

$$
\begin{aligned}
& \hat{\mathbf{x}}_{k+1}=\left[\begin{array}{llll}
\mathbf{0} & \mathbf{0} & \mathbf{0} & \mathbf{I}
\end{array}\right] \\
& {\left[\begin{array}{cccc}
\mathbf{F}_{k} \mathbf{P}_{k} \mathbf{F}_{k}^{T}+\mathbf{G}_{k} \mathbf{Q}_{k} \mathbf{G}_{k}^{T} & \mathbf{0} & \mathbf{0} & \mathbf{I} \\
\mathbf{0} & \mathbf{R}_{k+1} & \mathbf{0} & \mathbf{H}_{k+1} \\
\mathbf{0} & \mathbf{0} & \mathbf{0} & \mathbf{L}_{k+1} \\
\mathbf{I} & \mathbf{H}_{k+1}^{T} & \mathbf{L}_{k+1}^{T} & \mathbf{0}
\end{array}\right]^{+}} \\
& {\left[\begin{array}{c}
\mathbf{F}_{k} \hat{\mathbf{x}}_{k}+\mathbf{u}_{k} \\
\mathbf{y}_{k+1}-\mathbf{p}_{k+1} \\
-\mathbf{z}_{k+1} \\
\mathbf{0}
\end{array}\right]}
\end{aligned}
$$

$$
\mathbf{P}_{k+1}=-\left[\begin{array}{llll}
\mathbf{0} & \mathbf{0} & \mathbf{0} & \mathbf{I}
\end{array}\right]
$$$$
\left[\begin{array}{cccc}
\mathbf{F}_{k} \mathbf{P}_{k} \mathbf{F}_{k}^{T}+\mathbf{G}_{k} \mathbf{Q}_{k} \mathbf{G}_{k}^{T} & \mathbf{0} & \mathbf{0} & \mathbf{I} \\
\mathbf{0} & \mathbf{R}_{k+1} & \mathbf{0} & \mathbf{H}_{k+1} \\
\mathbf{0} & \mathbf{0} & \mathbf{0} & \mathbf{L}_{k+1} \\
\mathbf{I} & \mathbf{H}_{k+1}^{T} & \mathbf{L}_{k+1}^{T} & \mathbf{0}
\end{array}\right]^{+}
$$$$
\left[\begin{array}{l}
0 \\
\mathbf{0} \\
\mathbf{0} \\
\mathbf{I}
\end{array}\right]
$$

After the estimate $\hat{\mathbf{x}}_{k+1}$ is computed, it can be used to substitute for $\hat{\mathbf{x}}_{k+1}^{-}$, and the linearization process with respect to $\hat{\mathbf{x}}_{k+1}^{-}$may be conducted again. The convergent estimate $\hat{\mathbf{x}}_{k+1}$ is the final solution.

\section{Application to Attitude DETERMINATION}

For applying the ML-based filter for attitude determination, the attitude kinematics and the sensor measurements are described as follows.

\section{Attitude Kinematics}

In the following discussion, the attitude matrix is represented by the quaternion. The quaternion $\overline{\mathbf{q}}$ representing a rotation is defined as

$$
\overline{\mathbf{q}}=\left[\begin{array}{c}
q_{1} \\
q_{2} \\
q_{3} \\
q_{4}
\end{array}\right]=\left[\begin{array}{c}
\mathbf{q} \\
q_{4}
\end{array}\right]=\left[\begin{array}{c}
\mathbf{n} \sin (\theta / 2) \\
\cos (\theta / 2)
\end{array}\right],
$$

where $\mathbf{n}$ is a unit vector represents the axis of rotation; $\theta$ is the angle of rotation about the axis $\mathbf{n}$. The normalized quaternion will be denoted by an overbar. The quaternion possesses three degrees of freedom and satisfies the constraint

$$
\overline{\mathbf{q}}^{T} \overline{\mathbf{q}}=1
$$

The attitude matrix is obtained from the quaternion according to the relation [17]

$$
\mathbf{A}(\overline{\mathbf{q}})=\left(\left|q_{4}\right|^{2}-|\mathbf{q}|^{2}\right) \mathbf{I}+2 \mathbf{q} \mathbf{q}^{T}+2 q_{4}[|\mathbf{q}|]
$$

where $I$ is the $3 \times 3$ identity matrix and $[|q|]$ is the antisymmetric matrix defined as

$$
[|\mathbf{q}|]=\left[\begin{array}{ccc}
0 & q_{3} & -q_{2} \\
-q_{3} & 0 & q_{1} \\
q_{2} & -q_{1} & 0
\end{array}\right]
$$

This notation will be used generally for any $3 \times 3$ antisymmetric matrix generated from a three-vector. The attitude matrix defined above transforms the coordinates of vectors in the reference frame to those in the body frame.

The kinematic relation between the attitude representation and the angular velocity can be found [20] to be

$$
\frac{d}{d t} \overline{\mathbf{q}}(t)=\frac{1}{2} \Omega(\omega(t)) \overline{\mathbf{q}}(t),
$$

where

$$
\boldsymbol{\Omega}(\omega)=\left[\begin{array}{cccc}
0 & \omega_{3} & -\omega_{2} & \omega_{1} \\
-\omega_{3} & 0 & \omega_{1} & \omega_{2} \\
\omega_{2} & -\omega_{1} & 0 & \omega_{3} \\
-\omega_{1} & -\omega_{2} & -\omega_{3} & 0
\end{array}\right]
$$


and $\omega=\left[\begin{array}{lll}\omega_{1} & \omega_{2} & \omega_{3}\end{array}\right]$ denotes the angular velocity of the transformation. The equation (25) can also be written as

$$
\frac{d}{d t} \overline{\mathbf{q}}(t)=\frac{1}{2} \mathbf{\Xi}(\overline{\mathbf{q}}(t)) \omega(t)
$$

where

$$
\Xi(\overline{\mathbf{q}})=\left[\begin{array}{ccc}
q_{4} & -q_{3} & q_{2} \\
q_{3} & q_{4} & -q_{1} \\
-q_{2} & q_{1} & q_{4} \\
-q_{1} & -q_{2} & -q_{3}
\end{array}\right]
$$

Assuming that the angular velocity can be modeled as $\omega=\omega_{k}+\varepsilon_{\omega}$, where $\varepsilon_{\omega}$ is the zero-mean Gaussian white noise with covariance matrix $\mathbf{P}_{\omega}$, the continuous-time state equation of the attitude is given by

$$
\frac{d}{d t} \overline{\mathbf{q}}=\frac{1}{2} \Omega\left(\omega_{k}\right) \overline{\mathbf{q}}+\frac{1}{2} \boldsymbol{\Xi}(\overline{\mathbf{q}}) \varepsilon_{\omega}
$$

According to (29), the discrete-time model can be written as

$$
\overline{\mathbf{q}}_{k+1}=\mathcal{T}\left(t_{k+1}, t_{k}\right) \overline{\mathbf{q}}_{k}+\mathbf{v}_{k} .
$$

If the angular velocity $\omega_{k}$ is constant from time $t_{k}$ to $t_{k+1}$, the state transition matrix $\mathcal{T}\left(t_{k+1}, t_{k}\right)$ can be written as

$$
\mathcal{T}\left(t_{k+1}, t_{k}\right)=\exp \left(\frac{1}{2} \Omega\left(\omega_{k}\right) \cdot\left(t_{k+1}-t_{k}\right)\right) .
$$

The error vector $\mathbf{u}_{k}$ is a zero-mean white Gaussian noise with covariance matrix approximated by $\mathbf{Q}_{k}=$ $\frac{1}{4} \int_{t_{k}}^{t_{k+1}} \mathcal{T}\left(t_{k+1}, \tau\right) \Xi\left(\hat{\overline{\mathbf{q}}}_{k}\right) \mathbf{P}_{\omega} \boldsymbol{\Xi}^{T}\left(\hat{\overline{\mathbf{q}}}_{k}\right) \mathcal{T}^{T}\left(t_{k+1}, \tau\right) d \tau$.

\section{Sensor Measurements}

The attitude sensor measurements considered here are GPS carrier phase observables. In the following discussion, it is assumed that integer ambiguities have been solved and the phase observables are ambiguityfree. Based on the discussion in section II, the relation between the double-difference ambiguity-free phase observables $\Psi_{i}^{j}$ and the attitude $A$ is (cf.(4))

$$
\Psi_{i}^{j}\left(t_{k}\right)=\mathbf{b}_{i}^{T} \mathbf{A}\left(\overline{\mathbf{q}}\left(t_{k}\right)\right) \mathbf{s}^{j}+\mathbf{w}_{i}^{j}\left(t_{k}\right)
$$

where the vector $b_{i}$ is the $i$-th baseline vector represented in the body coordinate system; $\mathbf{s}^{j}$ equals to the difference between directional vector $\mathbf{g}^{j+1}$ and $\mathbf{g}^{1}$ represented in the reference coordinate system; $\Psi_{i}^{j}$ is the corresponding double-difference ambiguity-free phase measurements; the error vector $\mathbf{w}_{i}^{j}$ is the white noise with distribution $\mathcal{N}\left(\mathbf{0}, \mathbf{R}_{i}^{j}\right)$.

Based on (32), since

$$
\mathbf{b}_{i}^{T} \mathbf{A}(\overline{\mathbf{q}}) \mathbf{s}^{j}=\operatorname{Tr}\left(\mathbf{A}(\overline{\mathbf{q}})\left(\mathbf{B}_{i}^{j}\right)^{T}\right),
$$

where $\operatorname{Tr}$ denotes the trace operation and the matrix B is given by

$$
\mathbf{B}_{i j}=\mathbf{b}_{i}\left(\mathbf{s}^{j}\right)^{T} \text {. }
$$

Substituting (24) into (33), we have

$$
\begin{aligned}
\mathbf{b}_{i}^{T} \mathbf{A}(\overline{\mathbf{q}}) \mathbf{s}^{j}= & \left(q_{4}^{2}-\mathbf{q q}^{T}\right) \operatorname{Tr} \mathbf{B}_{i j}^{T}+2 \operatorname{Tr}\left(\mathbf{q q} \mathbf{q}^{T} \mathbf{B}_{i j}^{T}\right) \\
& +2 q_{4} \operatorname{Tr}\left([|\mathbf{q}|] \mathbf{B}_{i j}^{T}\right) .
\end{aligned}
$$

Introducing the quantities

$$
\begin{aligned}
\sigma_{i j} & =\operatorname{Tr} \mathbf{B}_{i j}=\mathbf{b}_{i}^{T} \mathbf{s}^{j}, \\
\mathbf{S}_{i j} & =\mathbf{B}_{i j}+\mathbf{B}_{i j}^{T}=\mathbf{b}_{i}\left(\mathbf{s}^{j}\right)^{T}+\mathbf{s}^{j} \mathbf{b}_{i}^{T}, \\
\mathbf{Z}_{i j} & =\mathbf{b}_{i} \times \mathbf{s}^{j},
\end{aligned}
$$

leads to the bilinear form

$$
\mathbf{b}_{i}^{T} \mathbf{A}(\overline{\mathbf{q}}) \mathbf{s}^{j}=\overline{\mathbf{q}}^{T} \mathbf{K}_{i j} \overline{\mathbf{q}}
$$

where the $4 \times 4$ matrix $\mathbf{K}_{i j}$ is defined by

$$
\mathbf{K}_{i j}=\left[\begin{array}{cc}
\mathbf{S}_{i j}-\sigma_{i j} \mathbf{I} & \mathbf{Z}_{i j} \\
\mathbf{Z}_{i j}^{T} & \sigma_{i j}
\end{array}\right]
$$

Thus, the sensor measurement equation (32) can be simplified as

$$
\boldsymbol{\Psi}_{i}^{j}\left(t_{k}\right)=\overline{\mathbf{q}}^{T}\left(t_{k}\right) \mathbf{K}_{i j}\left(t_{k}\right) \overline{\mathbf{q}}\left(t_{k}\right)+\mathbf{w}_{i}^{j}\left(t_{k}\right)
$$

Assuming that there are $m$ baseline vectors and $n$ satellites, the matrix form measurement equation can be written as

$$
\begin{aligned}
\boldsymbol{\Psi}_{k}= & {\left[\begin{array}{c}
\boldsymbol{\Psi}_{1}^{1} \\
\boldsymbol{\Psi}_{1}^{2} \\
\vdots \\
\boldsymbol{\Psi}_{m}^{n-1}
\end{array}\right] } \\
= & {\left[\begin{array}{cccc}
\overline{\mathbf{q}}^{T} & \mathbf{0} & \mathbf{0} & \mathbf{0} \\
\mathbf{0} & \overline{\mathbf{q}}^{T} & \mathbf{0} & \mathbf{0} \\
\mathbf{0} & 0 & \ddots & \mathbf{0} \\
\mathbf{0} & \mathbf{0} & \mathbf{0} & \overline{\mathbf{q}}^{T}
\end{array}\right]\left[\begin{array}{c}
\mathbf{K}_{11} \\
\mathbf{K}_{12} \\
\vdots \\
\mathbf{K}_{m(n-1)}
\end{array}\right] \overline{\mathbf{q}}+\mathbf{w}_{k} \cdot(4) }
\end{aligned}
$$

\section{Attitude Determination by ML-based Filter}

From the above discussion, it is shown that the measurement equation is nonlinear. In the following, the estimate of quaternion and the corresponding covariance matrix will be derived by the ML-based filtering.

According to the quaternion norm constraint and the equations (30) and (41), the linearized equations can be written as

$$
\begin{aligned}
\overline{\mathbf{q}}_{k+1} & =\mathcal{T}\left(t_{k+1}, t_{k}\right) \overline{\mathbf{q}}_{k}+\mathbf{v}_{k} \\
\boldsymbol{\Psi}\left(t_{k+1}\right) & =\mathbf{H}_{k+1} \overline{\mathbf{q}}_{k+1}+\mathbf{w}_{k+1}+\mathbf{p}_{k+1} \\
\mathbf{0} & =\mathbf{L}_{k+1} \overline{\mathbf{q}}_{k+1}+\mathbf{z}_{k+1}
\end{aligned}
$$


where

$$
\begin{aligned}
& \mathbf{H}_{k+1}=\left[\begin{array}{cccc}
\left(\hat{\overline{\mathbf{q}}}_{k+1}^{-}\right)^{T} & \mathbf{0} & \mathbf{0} & \mathbf{0} \\
\mathbf{0} & \left(\hat{\overline{\mathbf{q}}}_{k+1}^{-}\right)^{T} & \mathbf{0} & \mathbf{0} \\
\mathbf{0} & \mathbf{0} & \left(\hat{\overline{\mathbf{q}}}_{k+1}^{-}\right)^{T} & \mathbf{0} \\
\mathbf{0} & \mathbf{0} & \mathbf{0} & \left(\hat{\overline{\mathbf{q}}}_{k+1}^{-}\right)^{T}
\end{array}\right] . \\
& {\left[\begin{array}{c}
\mathbf{K}_{11} \\
\mathbf{K}_{12} \\
\vdots \\
\mathbf{K}_{m(n-1)}
\end{array}\right] \text {, }} \\
& \mathbf{p}_{k+1}=\left[\begin{array}{c}
\left(\hat{\overline{\mathbf{q}}}_{k+1}^{-}\right)^{T} \mathbf{K}_{11} \hat{\overline{\mathbf{q}}}_{k+1}^{-} \\
\left(\overline{\mathbf{q}}_{k+1}^{-}\right)^{T} \mathbf{K}_{12} \overline{\overline{\mathbf{q}}}_{k+1}^{-} \\
\vdots \\
\left(\hat{\overline{\mathbf{q}}}_{k+1}^{-}\right)^{T} \mathbf{K}_{m(n-1)} \hat{\overline{\mathbf{q}}}_{k+1}^{-}
\end{array}\right] \\
& -\mathrm{H}_{k+1} \hat{\overline{\mathbf{q}}}_{k+1}^{-} \text {, } \\
& \mathbf{L}_{k+1}=2\left(\hat{\overline{\mathrm{q}}}_{k+1}^{-}\right)^{T}, \\
& \mathbf{z}_{k+1}=-\left(\hat{\overline{\mathbf{q}}}_{k+1}^{-}\right)^{T} \hat{\overline{\mathrm{q}}}_{k+1}^{-}-1 \text {. }
\end{aligned}
$$

According to (21) and (22), the filtered estimate $\hat{\mathbf{q}}_{k+1}$ and the corresponding covariance matrix $\mathbf{P}_{k+1}$ can be computed. For satisfying the nonlinear constraint equation, $\hat{\overline{\mathbf{q}}}_{k+1}$ is being substituted for $\hat{\overline{\mathbf{q}}}_{k+1}^{-}$until the convergent $\hat{\overline{\mathbf{q}}}_{k+1}$ is found. The convergent condition is the $1 \%$ norm error, while the normalization is performed to satisfy the constraint in the next iteration.

\section{The Simulation}

To verify our filtering algorithm, the simulation has been conducted. In the simulation, the white noise was added on the carrier phase observables for assessing the performance of the proposed method while the platform being rotated. Three non-colinear antennae were assumed to get the GPS satellites' signals and could form two baseline vectors for attitude determination. Note that the carrier phase integer ambiguities were computed by the ambiguity function method, hence, the carrier phase observables are all ambiguity-free.

The configuration of the simulation is described below. Two aluminum bars with three antennae mounted at the ends was used to represent two baseline vectors. The configuration of the two baseline vectors are shown in Figure 2. According to the figure, the representation of $b_{1}$ in the body coordinate system was 5.885[ $\left.\begin{array}{lll}1 & 0 & 0\end{array}\right]$ and the representation of $\mathbf{b}_{2}$ is $2.99\left[\cos \left(19.3472^{\circ}\right) \sin \left(19.3472^{\circ}\right) \quad 0\right]$. These two aluminum bars were fixed on a rotation platform, which could be rotated by the AC servomotor.

The simulation is described as follows. The satellites ephemerides of PRN 1, 6, 14, 21, 22, 25, 29, 30 were

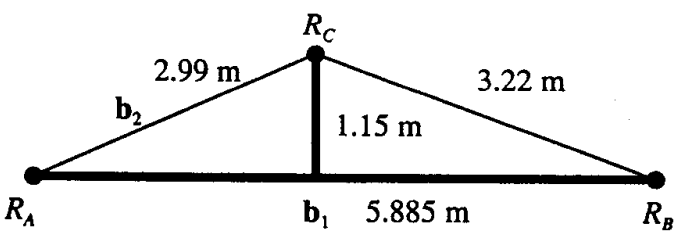

Figure 2: Configuration of three antennae for simulation and kinematic experiment

received by GPS receivers for computing the satellites' positions. The platform was assumed to be rotated with a constant rate $-0.0307 \mathrm{rad} / \mathrm{sec}$. The initial attitude matrix was given as

$$
\mathbf{A}_{\text {ini }}=\left[\begin{array}{ccc}
0.4512 & 0.7282 & -0.5159 \\
-0.6554 & -0.1221 & -0.7454 \\
-0.6057 & 0.6744 & 0.4221
\end{array}\right] .
$$

Based on the above parameters, the phase observables can be generated for the simulation. The simulation time was 200 seconds. The white noise $\mathcal{N}\left(0,(0.01 \lambda)^{2}\right)$ is added on the phase measurements to assess the performance of the proposed algorithm. The generated phase measurements are shown in Figures 3 and 4.

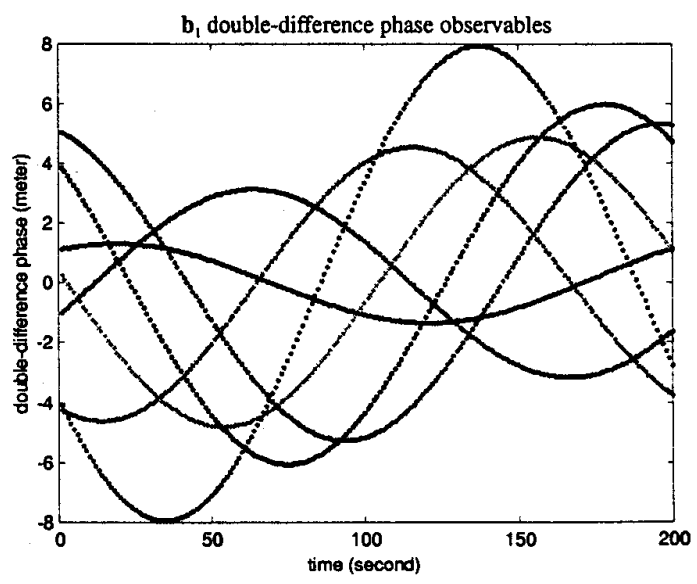

Figure 3: $b_{1}$ double-difference phase observables in simulation

The filtered attitude matrix computed by the proposed method and the unfiltered attitude calculated by the QUEST algorithm via the measurement of a single epoch is then transferred to yaw, roll, pitch angles, which are shown in Figures 5, 6, and 7. The expressions for these rotation angles in terms of the elements of the attitude matrix $\mathbf{A}$ are [20]

$$
\text { pitch }=\arcsin \left(\mathbf{A}_{23}\right)
$$




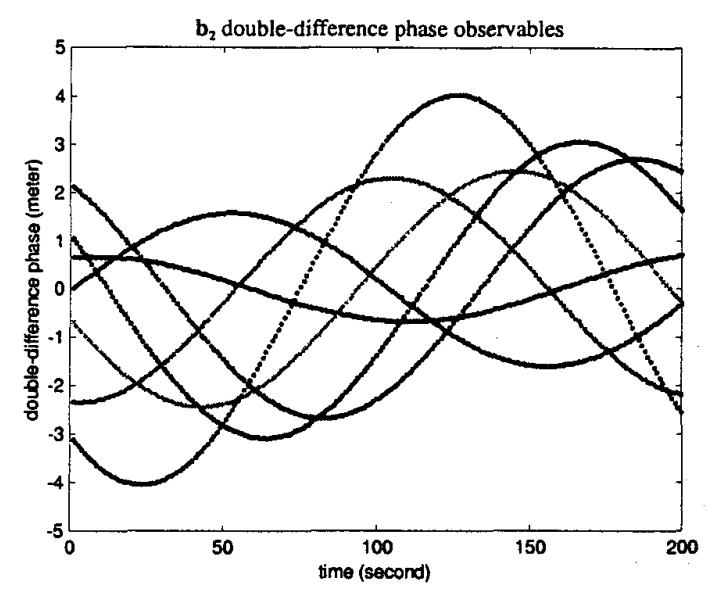

Figure 4: $\mathbf{b}_{2}$ double-difference phase observables in simulation

$$
\begin{aligned}
\text { yaw } & =-\arctan \left(\mathbf{A}_{21} / \mathbf{A}_{22}\right) \\
\text { roll } & =-\arctan \left(\mathbf{A}_{13} / \mathbf{A}_{33}\right) .
\end{aligned}
$$

From the simulation results, it can be shown that the proposed method can be used to determine attitudes in the kinematic case.

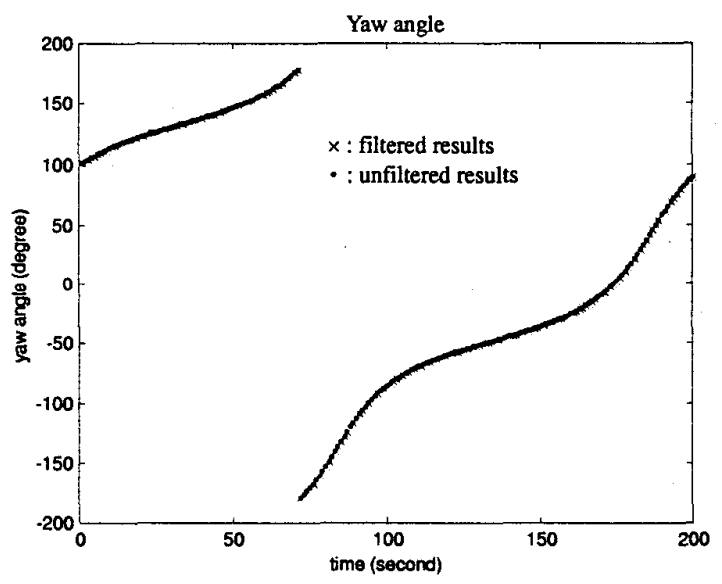

Figure 5: Yaw angle of attitude matrix in simulation

\section{CONCLUSION}

This paper is concentrated on attitude determination via GPS carrier phase observables. Two main issues are considered in this topic. The first part is the ambiguity resolution, while the ambiguity function method is adopted to resolve cycle ambiguities. The second part is the algorithm for attitude determination. Based on



Figure 6: Roll angle of attitude matrix in simulation

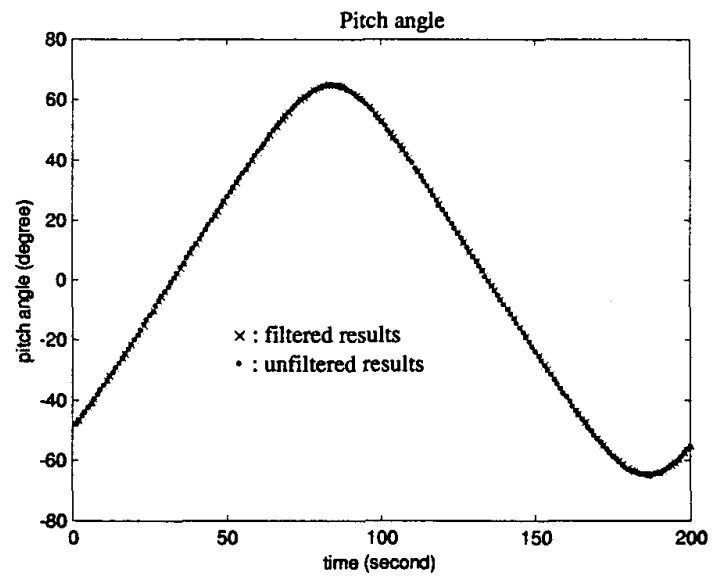

Figure 7: Pitch angle of attitude matrix in simulation

the concept of maximum-likelihood estimation, the MLbased filtering using GPS carrier phase measurements for attitude determination has been studied. Some preliminaries about the maximum likelihood estimation are reviewed. The nonlinear constraints on state variable are also considered. This algorithm can be used to estimate the state variable with some specific constraint equation. If the considered system is nonlinear, the linearized model is used. Comparing with the application in attitude determination, the norm of quaternion is the constraint equation. The sensor measurements observed from GPS are also nonlinear functions with respect to state variable, quaternion. For verifying the proposed algorithm, the simulation has been conducted. The simulation can be used to assess the performance of the proposed algorithm. The simulation results showed that the filtered results were smoother than the unfiltered results which were directly computed by GPS at every epoch. 


\section{References}

[1] I. Y. Bar-Itzhack. Request, a recursive quest algorithm for sequential attitude determination. Journal of Guidance, Control, and Dynamics, 19(5):1034-1038, 1996.

[2] R. Brown and P. Ward. A gps receiver with builtin precision pointing capability. In IEEE Position Location and Navigation Symposium, pages 83-93, Las Vegas, Nevada, March 1990.

[3] S. L. Campbell and C. D. Meyer. Generalized Inverse of Linear Transformations. Pitman, London, 1979.

[4] Dingsheng Chen. Development of a Fast Ambiguity Search Filtering (FASF) Method for GPS Carrier Phase Ambiguity Resolution. PhD thesis, The University of Calgary, Calgary, Alberta, December 1994.

[5] Clark Emerson Cohen. Attitude Determination Using GPS: Development of an All Solid-State Guidance, Navigation, and Control Sensor for Air and Space Vehicles Based on the Global Positioning System. PhD thesis, Stanford University, December 1992.

[6] Bernhard Hofmann-Wellenhof, Herbert Lichtenegger, and James Collins. Global Positioning System Theory and Practice. Springer-Verlag Wien, New York, fourth revised edition, 1997.

[7] Elliott D. Kaplan, editor. Understanding GPS: Principles and Applications. Artech House, Boston London, 1996.

[8] E. J. Lefferts, F. L. Markley, and M. D. Shuster. Kalman filtering for spacecraft attitude estimation. Journal of Guidance, Control and Dynamics, 5(5):417-429, September-October 1982.

[9] S. J Leon. Linear Algebra with Applications. Macmillan College Publishing Company, fourth edition, 1994.

[10] Gang Lu. Development of a GPS Multi-Antenna System for Attitude Determination. PhD thesis, The University of Calgary, Calgary, Alberta, January 1995.

[11] F. L. Markley, N. Berman, and U. Shaked. Deterministic ekf-like estimator for spacecraft attitude estimation. In Proceedings of the American Control Conference, pages 247-251, Baltimore, Maryland, June 1994.
[12] Ramine Nikoukhah, Alan S. Willsky, and Bernard C. Levy. Kalman filtering and riccati equations for descriptor systems. IEEE Transations on Automatic Control, 37(9):1325-1342, September 1992.

[13] B. W. Remondi. Using the Global Positioning System (GPS) Phase Observable for Relative Geodesy: Modelling, Processing, and Results. PhD thesis, University of Texas, Austin, May 1984.

[14] B. W. Remondi. Performing centimeter-level surveys in seconds with gps carrier phase: Initial results. In Proceedings of the Fourth International Geodetic Symposium on Satellite Positioning, pages 1229-1249, Austin, Texas, April 1986.

[15] M. D. Shuster. A simple kalman filter and smoother for spacecraft attitude. Journal of the Astronautical Sciences, 37(1):88-106, 1989.

[16] M. D. Shuster. Kalman filtering of spacecraft attitude and the quest model. Journal of the Astronautical Sciences, 38(3):377-393, July-September 1990.

[17] M. D. Shuster. Survey of attitude representations. Journal of the Astronautical Sciences, pages 439 517 , October 1993.

[18] M. D. Shuster and S. D. Oh. Three-axis attitude determination from vector observations. Journal of Guidance, Control and Dynamics, 4(1):70-77, January-February 1981.

[19] P. J. G. Teunissen. The least-square ambiguity decorrelation adjustment: A method for fast gps integer ambiguity estimation. Journal of Geodesy, $70(1-2): 65-82,1995$.

[20] James R. Wertz, editor. Spacecraft Attitude Determination and Control. Kluwer Academic Publishers, 1978.

[21] Joz Wu and Fong-Gee Yiu. Cosine functions of gps carrier phases for parameter estimation. Journal of Surveying Engineering, 123(3):113-125, August 1997. 